\title{
Gelsolin Can Be a Prognostic Biomarker and Correlated with Immune Infiltrates in Gastric Cancer
}

\author{
Yingmei $\mathrm{Wu} \mathbb{1}^{1,2, *}$ \\ Junhui Zheng ${ }^{1,2, *}$ \\ Yanhua Yan ${ }^{1,2}$ \\ Jiduo Liu $^{3}$ \\ Yingchun Zhou ${ }^{3}$
}

'Guangzhou University of Chinese Medicine, Guangzhou, 510000 , People's Republic of China; ${ }^{2}$ Lingnan Medical Research Center, Guangzhou University of Chinese Medicine, Guangzhou, 510000 , People's Republic of China; ${ }^{3}$ Department of Clinical Laboratory, The First Affiliated Hospital, Guangzhou University of Chinese Medicine, Guangzhou, 510000, People's Republic of China

*These authors contributed equally to this work
Correspondence: Yingchun Zhou; Jiduo Liu

Department of Clinical Laboratory, The First Affiliated Hospital, Guangzhou University of Chinese Medicine, Guangzhou, 510000, People's Republic of China

Email yingchunbaby@I26.com;

13668996592@163.com
Background: Gelsolin (GSN) is the most widely expressed actin-severing protein in humans, which could regulate cell morphology, differentiation, movement and apoptosis. This study aims to explore the GSN as a prognostic biomarker of stomach adenocarcinoma (STAD).

Methods: In this study, we used several online databases to comprehensively analyze the role of GSN in STAD. Oncomine and HPA databases were used to explore the GSN expression in various cancer, especially in gastric cancer. Then, UALCAN database was used to evaluate the relationship between GSN expression and promoter methylation in clinical characteristics. Finally, we used TIMER to analyze the correlation between GSN expression and immune infiltrates in gastric cancer.

Results: GSN was down-regulated in gastric cancer, and decreased expression of GSN was related to worse survival. The GSN expression was significantly related to tumor purity in STAD and significantly correlated with infiltrating level of various immune cells, especially the dendritic cells.

Conclusion: Our study proposes that GSN can be served as the biomarker of disease and neoantigen for STAD treatment, which can improve the deficiency of disease-specific targeted therapies currently exist.

Keywords: gelsolin, gastric cancer, prognosis, immune cell infiltration

\section{Introduction}

Gastric cancer (GC) remains an important cause of cancer-related incidence and mortality, with over one million new cases and close to 800,000 deaths in 2018 . Therefore, it has been supposed to the fifth most frequently diagnosed cancer and the third-leading cause of cancer death. ${ }^{1}$ It is estimated that there were $1,033,701$ new cases of GC (representing 5.7\% of all cancer cases diagnosed) and 782,685 deaths related to GC in 2018 around the world. ${ }^{2}$ Furthermore, GC was the fourth most commonly diagnosed cancer type in men and the seventh-most commonly diagnosed cancer type in women. ${ }^{3}$ Although a recent global study indicated that compared to incidence, the mortality rates of GC have decreased more rapidly because of socioeconomic development, diagnosis improvement and treatment facilities amelioration. ${ }^{4}$ However, the mortality rate of GC is still high because of the difficulty in detection and asymptomatic in early stage. ${ }^{5}$

The early diagnosis plays a key role in curing GC while the unobvious symptom and the difficulties lying in the early diagnosis largely affect the effect of the 
treatment. Thus, the five-years survival rate of GC ranges between $20 \%$ and $40 \%$ in most countries. ${ }^{6,7}$ Meanwhile, because of the genetic complexity and heterogeneity, the traditional therapy, whether chemotherapy or anti-human epidermal growth factor receptor-2 (HER-2) therapy, ${ }^{8}$ having much to desire.

Gelsolin (GSN) belongs to the actin-binding proteins, a family which can mediate multiple cellular functions like cell motility, apoptosis, morphogenesis, and actin cytoskeletal remodeling. The most extensive features of gelsolin are its actin filament severing, capping, uncapping, and nucleating activities. ${ }^{9,10}$ Previous literature has addressed the role of GSN in cancer, many of this suggested that GSN functions as both tumor suppressor gene and oncogene. In other words, GSN will probably become a new mark in diagnoses and target in therapies. ${ }^{6}$ However, there were some reports that the therapeutic applicability of GSN depends on the cancer subtype. In Ras-induced tumors, it was shown that mutated GSN can suppress tumor growth. ${ }^{11}$ And research has suggested that high GSN expression under conditions such as heart disease, aging, and stress, all those characters are lethal for heart disease. ${ }^{12}$ In addition, previous studies show that the expression of GSN in colorectal cancer cells was necessary for invasion. ${ }^{13}$ Another report observed that the total GSN expression affects cell proliferation and viability by vitro techniques in head and neck squamous cell carcinoma. ${ }^{14}$ Recently, some case reports even found that a patient encounters renal gelsolin amyloidosis with the $p$. Asp174Asn mutation in the GSN gene, another with the phenotype of amyloidosis of the Finnish type because of GSN variant. ${ }^{15,16}$

In this study, we used several online datasets to analyze GSN expression and its relevance to prognosis in patients with GC, as well as the correlation with immune infiltration in the tumor microenvironment. Therefore, we proposed that GSN can be considered as a potential biomarker of GC occurrence and progression. Further, due to the limited number of disease-specific targeted therapies, GSN may be a promising neoantigen for STAD treatment.

\section{Materials and Methods}

\section{Oncomine and HPA Database Analysis}

Oncomine (https://www.oncomine.org/resource/login.html) ${ }^{17}$ and the human protein atlas (https://www.proteinatlas.org) $)^{18}$, are the databases we always used to identify the gene expression in diverse cancers. The threshold in Oncomine database is as follows: $P$-value of 0.001 , fold change of 1.5 , and gene ranking of all.

\section{TIMER Database Analysis}

The expression of GSN in various cancers and the relevance to the expression of GSN and the abundance of immune invasion were analyzed by the TIMER (https:// cistrome.shinyapps.io/timer/) $^{19}$, an online database that can analyze immune infiltrates in various cancers, comprehensively, systematically and respectively. GSN expression and tumor infiltrating immune cell gene markers by the related modules.

\section{UALCAN Database Analysis}

In order to study healthy tissue as well as tumor in different stage, grade and other clinicopathological characteristics, we used UALCAN (http://ualcan.path.uab.edu) ${ }^{20}$ to drawn 9 box plots. UALCAN is a database that includes 31 cancer types from the Cancer Genome Atlas (TCGA) clinical data.

\section{Kaplan-Meier Plotter Database Analysis}

Prognosis is usually comprised of overall survival (OS), First progression (FS), and post progression survival (PPS), we use Kaplan-Meier (https://kmplot.com/21 to construct survival curve and analyze correlative data. All data provide the hazard ratio (HR) value with $95 \%$ confidence intervals and log-rank P-values.

\section{Cell Culture and Quantitative Real-Time Polymerase Chain Reactions (qRT-PCR)}

Human gastric cancer cells and human normal gastric epithelial cell line GES-1 were acquired from the American Type Culture Collection (ATCC, Manassas, VA, USA). We placed the cells in RPMI-1640 Medium (Gibco, USA, No.8121571) supplemented with $10 \%$ fetal bovine serum (Gibco, USA, No.25200-056), cultivated it in $5 \% \mathrm{CO} 2$ incubator at $37^{\circ} \mathrm{C}$. We adopted the third generation of cell after revived the same batch of frozen cells, in order to ensure the stability of cell characteristics. TRIzol reagent (Life Technologies, USA, No.252604) lyses cells, then collect total RNA to reverse transcription (Evo M-MLVRT Premix Accurate Biotechnology, China, No. AG11706) and quantitative realtime PCR (qRT-PCR) analysis use SYBR Green (Accurate Biotechnology, No. AG11701) for quantification. The primers used in our study were as follows: GSN forward primer, 5'-GGTGTGGCATCAGGATTCAAG-3'; GSN reverse 
primer,3'-TTTCATACCGATTGCTGTTGGA-5'; GAPDH forward primer, 5'-GGACCTGACCTGCCGTCTAG-3'; GAPDH reverse primer, 3'-GTAGCCCAGGATGCC CTTGA-5' (Generay Biotech Co, Shanghai).

\section{Statistical Analysis}

The cut-off value of GSN expression is medium among the statistics. P-value, fold change, and mRNA were used to analyze gene expression in the Oncomine database, and the Kaplan Meier plots database act as tool to draw survival curves, all data calculated by $\mathrm{HR}$ and $P$-values from a $\mathrm{Log}$ rank test. In TIMER, we choose Spearman correlation analysis to evaluate. $\mathrm{P}<0.05$ was considered statistically significant. The GSN expression was calculated based on 2 $-\Delta \Delta \mathrm{Ct}$ method with GAPDH as an internal reference.

\section{Results}

\section{The Expression Levels of GSN in Various Types of Human Cancer}

We used three independent databases to analyze GSN expression between tumors and normal tissues. Firstly, the Oncomine database shows that GSN expression was down- regulated in bladder, breast, colorectal, esophageal, gastric, head and neck, lung, myeloma, ovarian, and prostate cancer compared to the normal tissues (Figure 1A). Meanwhile, we use TIMER to analyze the expression of GSN in pan-cancers between the tumor and adjacent normal tissues. GSN expression was significantly lower in BLCA (bladder urothelial carcinoma), BRCA (breast invasive carcinoma) and COAD (colon adenocarcinoma), ESCA (esophageal carcinoma), KICH (kidney chromophobe), KIRP (kidney renal papillary cell carcinoma), LIHC (liver hepatocellular carcinoma), LUA (lung adenocarcinoma), STAD (stomach adenocarcinoma), and UCEC (uterine corpus endometrial carcinoma) (Figure 1B). Further analysis from HPA website displays the GSN protein expression in different cancers. The results showed that the GSN protein is moderately expressed in STAD among various cancers (Figure 1C).

\section{Relationship Between GSN Expression and Clinical Characteristics in GC Patients}

We assessed whether GSN expression was associated with clinicopathological factors in patients with GC (Figure 2A). The results in GEPIA database are in excellent agreement
A

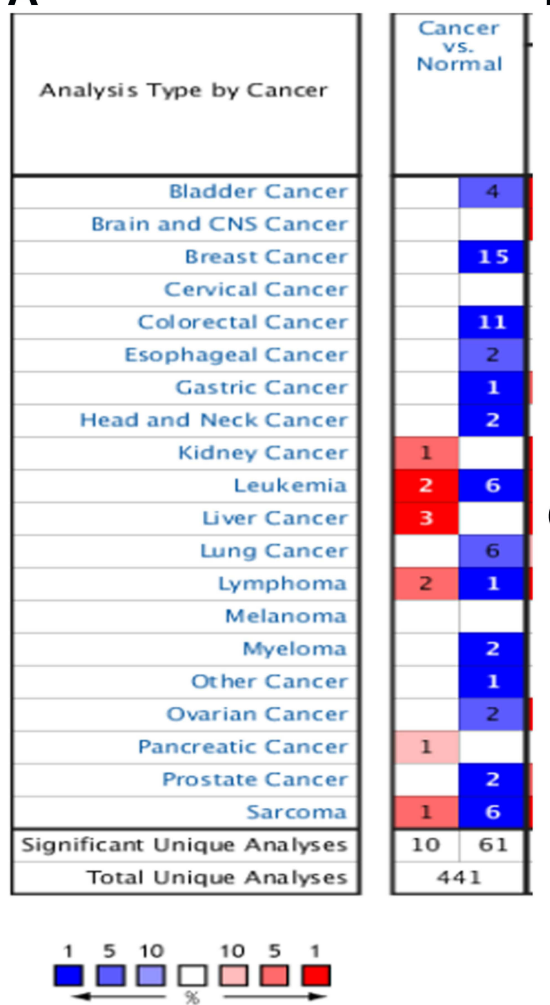

B
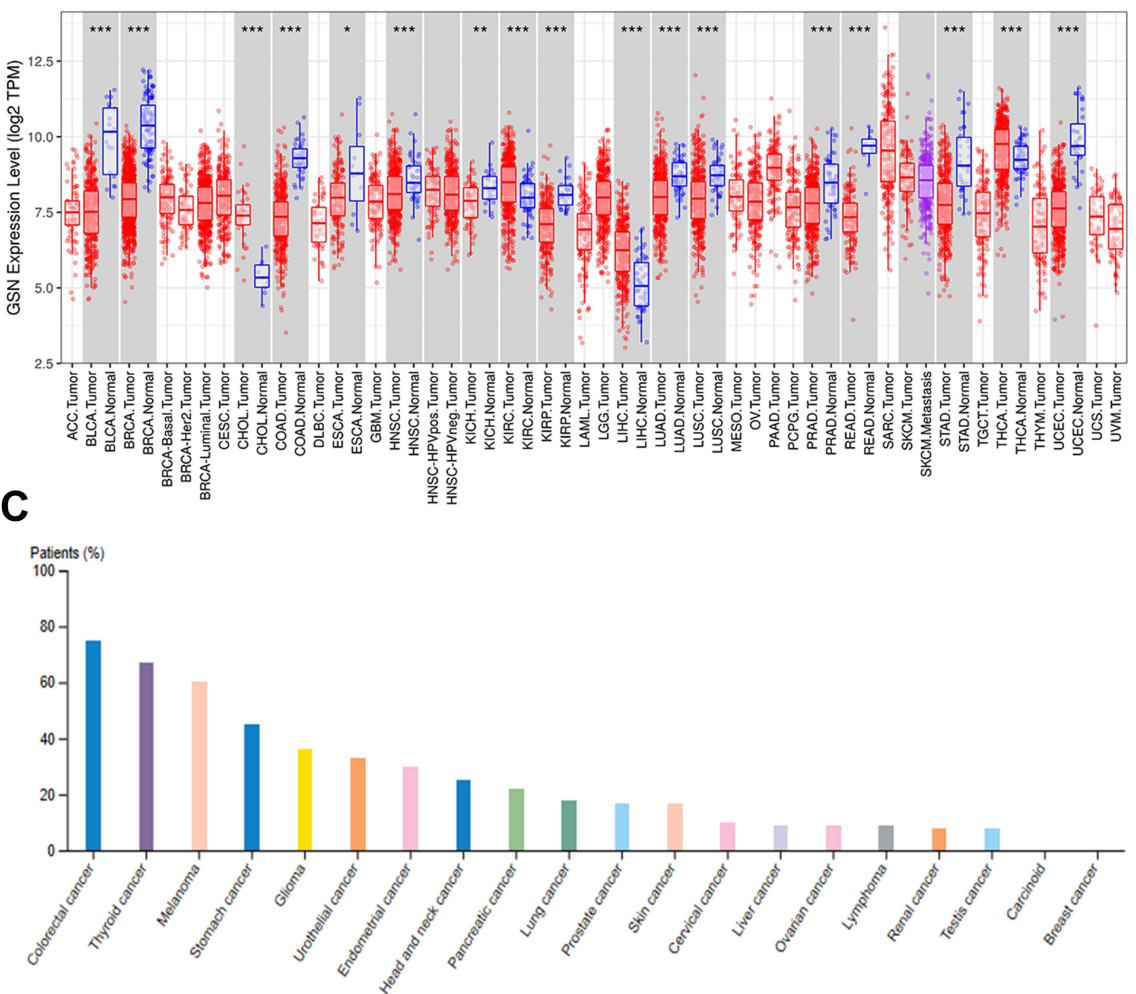

Figure I The expression levels of GSN in various types of human cancer. (A-C), the results are from Oncomine, TIMER, and HPA. P-value significant codes: $0 \leq * * * 0.001 \leq * * \leq 0.01 \leq * \leq 0.05$. 


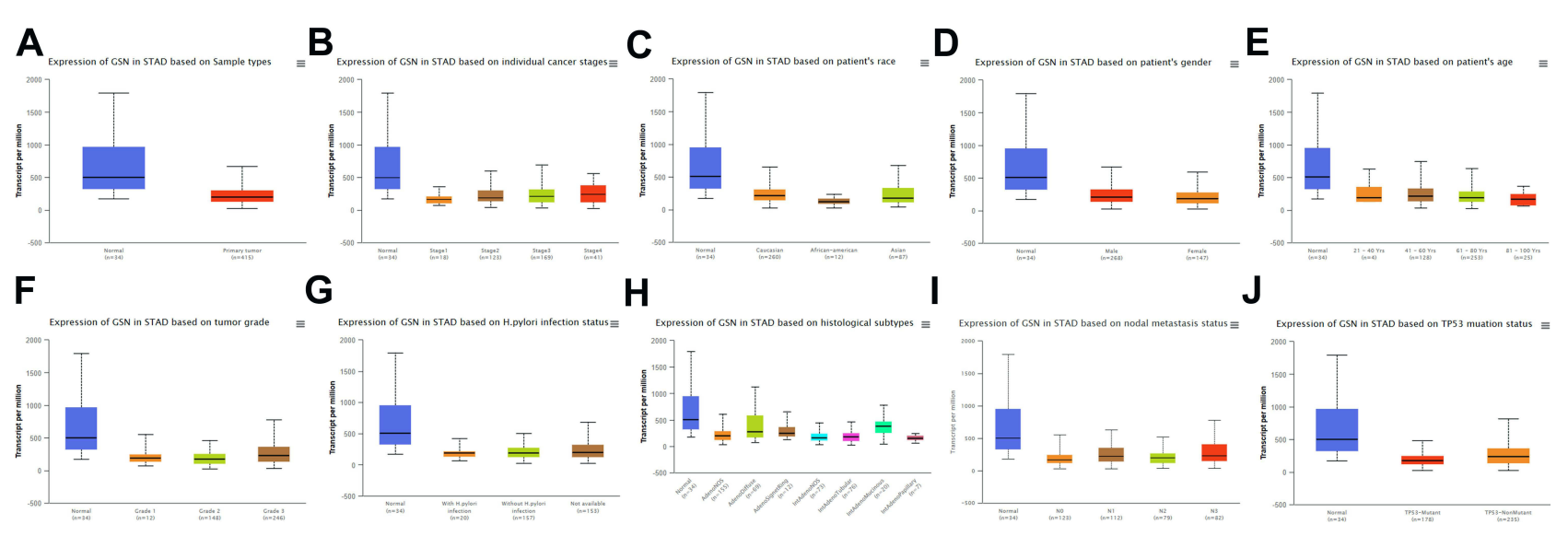

Figure 2 Relationship between GSN expression and clinical characteristics in GC patients. (A-J), expression of GSN on cancer stages, race, gender, age, tumor grade, $\mathrm{H}$ pylori infection status, histological subtypes, metastasis, and TP53 mutation.

with the previous paper. The tumor stage (Stage 1, Stage 2, Stage 3 and Stage 4), race (Caucasian, African and Asian), gender (male and female), age (41-60, 61-80, and 81-100 years old), tumor grade (Grade 1, Grade 2 and Grade 3), H. pylori infection, histological (AdenoNos, AdenoDiffuse, AdenosignetRing, IntAdenoNos, IntAdenoMucinous, IntAdenopapillary), metastasis (N0, N1, N2, N3), and TP53 mutation (Figure 2B-J), all of the changes has made aspects of GSN.

\section{Prognostic Potential of GSN in GC}

To investigate the correlation between GSN expression and survival rates, the survival curve and the impact were analyzed by the Kaplan-Meier plotter. Notably, GSN expression significantly impacts prognosis in STAD. Higher GSN expression, and poorer prognosis. $(\mathrm{OS}, \mathrm{HR}=0.73(0.59-0.91), \mathrm{P}=0.0045 . \mathrm{FP}, \mathrm{HR}=0.8$ (0.66-0.91), $\mathrm{P}=0.018$. PPS, HR $=0.76(0.6-0.96), \mathrm{P}=$
0.021) (Figure 3A-C). There are 2 gene symbols in Kaplan-Meier plotter, 200696_s_at and 214040_s_at, but the result of $200696 \_$s_at is contrary to expectation, so we delete it. This may be due to insufficient data, possibly characteristic of mucosal immunity. Anyhow, the prognostic valuation of GSN will be plainly obvious. Then we show the correlation between GSN expression and some clinicopathological factors via Table 1, these results indicated that the abnormal GSN and low survival rates may be more common in female and the late stage.

\section{GSN Expression is Correlated with Immune Infiltration Level in GC}

We explored the correlation between GSN expression and immune infiltration levels in STAD. The results showed that the GSN expression was significantly correlated with immune infiltration levels of various
A

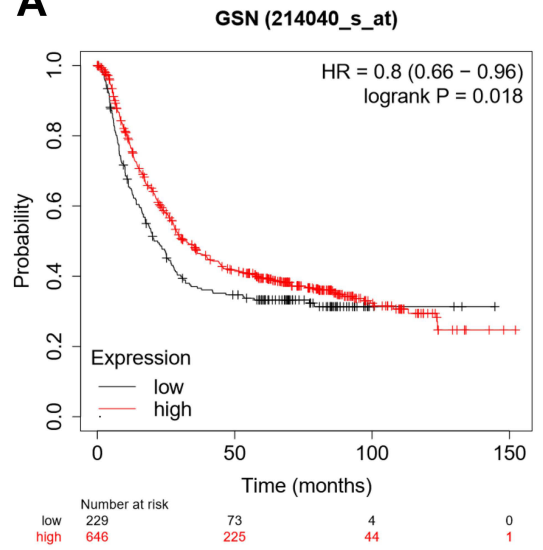

B

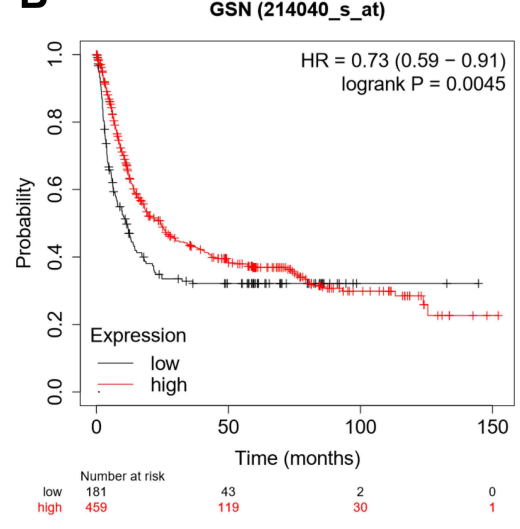

C GSN (214040_s_at)

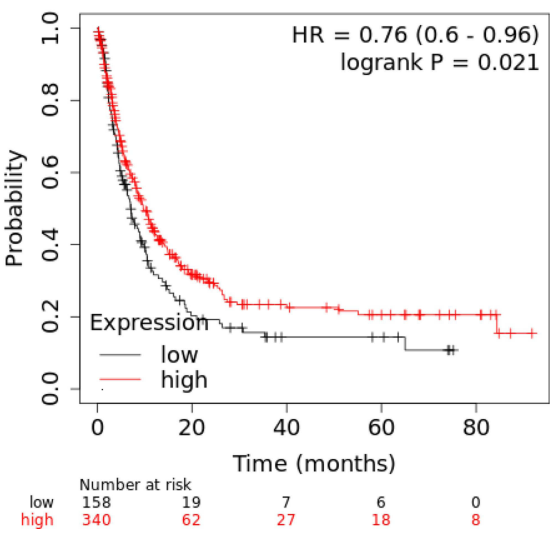

Figure 3 Prognostic potential of GSN in GC. (A), OS; (B), FP; (C), PPS. 
Table I The Correlation Between GSN Expression and Prognosis in GC with Respect to Clinicopathological Factors

\begin{tabular}{|c|c|c|c|c|c|c|}
\hline \multirow[t]{2}{*}{ Clinicopathological } & \multicolumn{3}{|c|}{ Overall Survival } & \multicolumn{3}{|c|}{ Progression-Free Survival } \\
\hline & $\mathbf{N}$ & Hazard Ratio & P-value & $\mathbf{N}$ & Hazard Ratio & P-value \\
\hline \multicolumn{7}{|l|}{ SEX } \\
\hline Female & 244 & $1.58(1.1-2.27)$ & 0.013 & 201 & $1.29(0.86-1.92)$ & 0.21 \\
\hline Male & 566 & $1.2(0.96-1.49)$ & 0.11 & 437 & & \\
\hline \multicolumn{7}{|l|}{ STAGE } \\
\hline I & 67 & $1.92(0.72-5.15)$ & 0.19 & 60 & $3.14(0.4 I-24.33)$ & 0.25 \\
\hline 2 & 140 & $2.11(1.16-3.86)$ & 0.013 & $|3|$ & $1.44(0.79-2.63)$ & 0.23 \\
\hline 3 & 305 & $1.41(1.05-1.88)$ & 0.021 & 186 & $1.26(0.84-1.9)$ & 0.26 \\
\hline 4 & 148 & $1.88(1.17-3.04)$ & 0.0086 & 141 & $1.74(1.06-2.84)$ & 0.026 \\
\hline \multicolumn{7}{|l|}{ STAGE T } \\
\hline 2 & $24 I$ & $\mathrm{I} .45(0.87-2.4 \mathrm{I})$ & 0.15 & 239 & $1.32(0.79-2.18)$ & 0.29 \\
\hline 3 & 204 & $1.55(I .08-2.2 I)$ & 0.015 & 204 & $1.54(1.1-2.18)$ & 0.013 \\
\hline 4 & 38 & $2.4 I(I-5.82)$ & 0.045 & 39 & $2.29(1.0 \mathrm{I}-5.2)$ & 0.043 \\
\hline \multicolumn{7}{|l|}{ STAGE N } \\
\hline 0 & 74 & $3.53(1.04-11.94)$ & 0.031 & 72 & $3.48(1.03-11.79)$ & 0.033 \\
\hline 1 & 225 & $1.34(0.89-2.02)$ & 0.16 & 222 & $1.28(0.87-1.89)$ & 0.21 \\
\hline 2 & 121 & $1.38(0.85-2.25)$ & 0.19 & 125 & $1.26(0.79-2.02)$ & 0.33 \\
\hline 3 & 76 & $3.28(1.46-7.4)$ & 0.0026 & 76 & $2.64(1.17-5.97)$ & 0.016 \\
\hline $1+2+3$ & 422 & $1.48(1.12-1.95)$ & 0.0056 & 423 & $1.35(1.05-1.74)$ & 0.021 \\
\hline \multicolumn{7}{|l|}{ STAGE M } \\
\hline 0 & 444 & $1.47(1.11-1.93)$ & 0.0063 & 443 & $1.4(1.07-1.82)$ & 0.013 \\
\hline I & 56 & $2.5(1.14-5.49)$ & 0.019 & 56 & $2.24(1.01-4.97)$ & 0.042 \\
\hline \multicolumn{7}{|c|}{ LAUREN CLASSIFICATION } \\
\hline Intestinal & 320 & $1.44(1.05-1.99)$ & 0.023 & 263 & $1.29(0.85-1.94)$ & 0.22 \\
\hline Diffuse & $24 I$ & $1.67(1.17-2.4)$ & 0.0048 & 231 & $1.78(1.24-2.55)$ & 0.0015 \\
\hline \multicolumn{7}{|l|}{ DIFFERENTIATIN } \\
\hline Poor & 165 & $0.83(0.56-1.24)$ & 0.36 & 121 & $1.66(0.93-2.98)$ & 0.084 \\
\hline
\end{tabular}

Abbreviation: $\mathrm{N}$, number.

immune cells in STAD. Concretely, GSN was significantly correlated with infiltration level of B cell, CD8+ $\mathrm{T}$ cells, CD4 $+\mathrm{T}$ cells, macrophages, neutrophil and dendritic cells in TME (Figure 4A-C). Moreover, GSN was significantly correlated with tumor purity in STAD. Above all, the expression of GSN is associated with immune regulation disorder (Table 2).

\section{GSN Expression in Different Gastric Cancer Cell Lines}

Data results generated from the qRT-PCR analysis shows that GSN was down-regulated in several human gastric carcinoma cell lines. For Figure 5 expression, the BGC-823 was shown to be the lowest level, and after it the MKN-45 AGS and MGC-803. $(\mathrm{P}<0.01)$. However, the specific reasons need further research.

\section{Discussion}

In this study, we describe the expression of GSN and its prognostic value in GC, and summarize the relevant knowledge about the immune features in gastric cancer. In short, GSN was down-regulated in GC tissues, and high expression GSN was related to longer survival. The GSN expression is related to tumor purity in STAD and significantly correlated with immune cell infiltration, especially the dendritic cells.

Understanding the mechanism of the gastric mucosal immunity in the development of gastric cancer may help us to search for new clinical diagnostic and therapeutic methods. ${ }^{22}$ In the human body, gastrointestinal mucosal is an important immune organ, ${ }^{23}$ which can play its immune function in a layer-by-layer progressive mode through innate and adaptive immunity ${ }^{24}$ and maintain the balance of microbe in an immune homeostasis mechanism. ${ }^{25}$ However, 

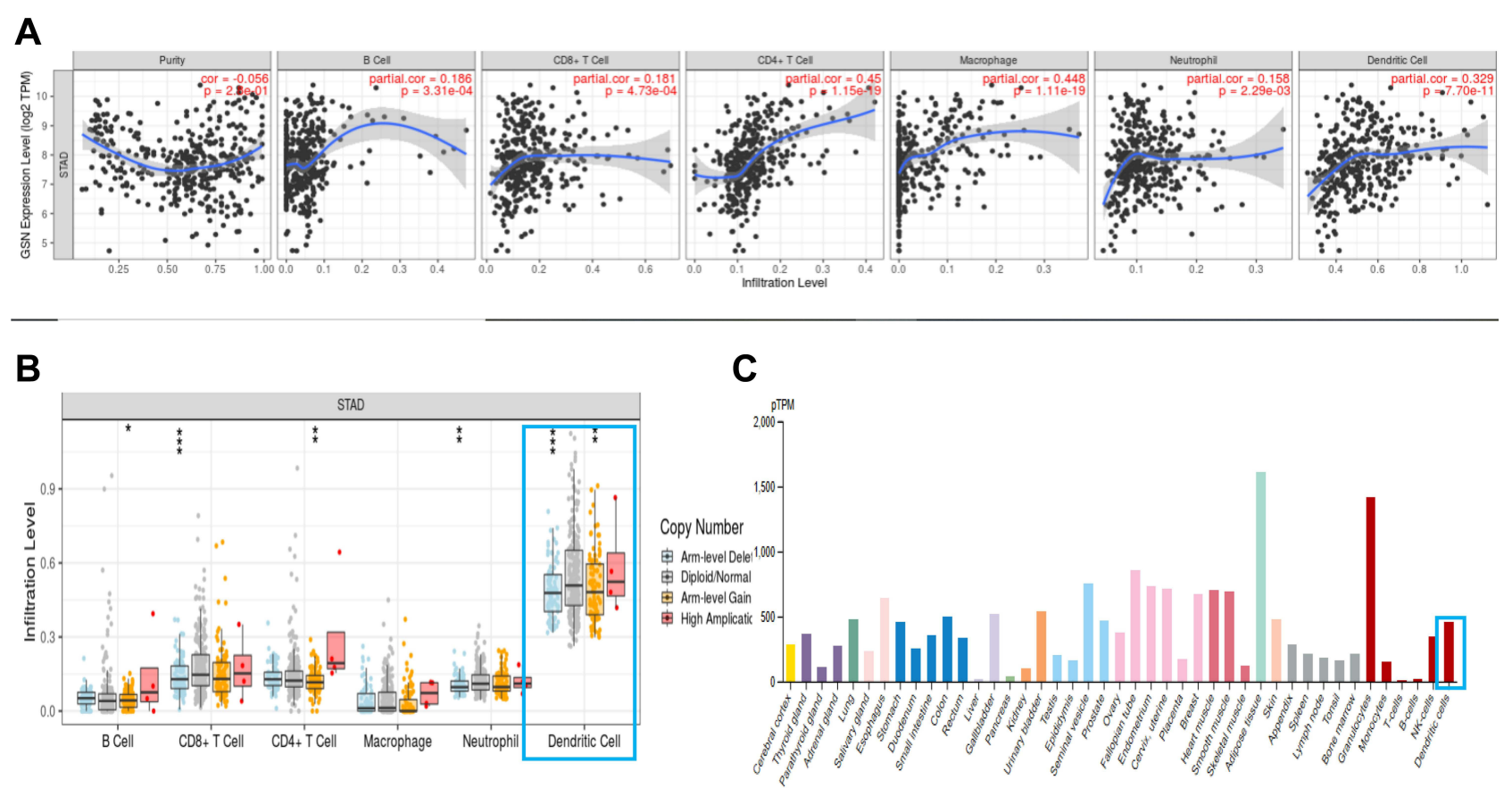

Figure 4 The correlation between GSN expression and immune infiltration level in GC. (A and $\mathbf{B}$ ), the result from TIMER database. (C), the immunoreaction of GSN from HPA database. P-value significant codes:0 $\leq * * * \leq 0.001 \leq * * \leq 0.01 \leq * \leq 0.05$.

the DCs immune response characters in human gastric mucosa were reported up to $2010 .^{26}$ After affected by pathogen-associated molecular patterns, DCs activate and maturate, only in this way can DCs play the initial immune response to pathogens. ${ }^{27-29}$ As the most potent antigen presenting cells (APC) and critical regulators of immune responses, which possessing the unique capacity to trigger primary adaptive immune responses through the antigenspecific activation of naive T cells. ${ }^{30}$ Before the pathogens pass APCs, immune response even cannot be activated quickly, ${ }^{31}$ However, the modulation of DC leads the tumor immune evasion in tumors and tumor-associated cells/factors in the TME and exhibit impaired or defective function, thus might mediate immunosuppression instead. ${ }^{32}$ Thus, our review identified the DCs character in GC environment via explore the expression levels of GSN. GSN, a protein highly expressed in murine and human hearts, which can regulate dynamic actin filament organization, regulate calcium actinsevering and capping. ${ }^{33,34}$ Previous literature has shown that GSN is an important character in cardio myoblast cells, which can reduce p-Akt and increase HIF-1a during hypoxia conditions to regulate apoptosis. Compared to wild-type littermates (GSN+/ +), GSN knockout mice (GSN-/-) were found to have lower mortality, markedly reduced hypertrophy, smaller late infarct size, less interstitial fibrosis, and improved cardiac function. ${ }^{12}$ In addition, $\mathrm{p}-\mathrm{GSN}$ can also bind to proinflammatory and bioactive molecules, including sphingosine 1-phosphate (S1P), lipopolysaccharide, and lysophosphatidic acid, which could temper host inflammatory responses during certain disease progression. Literatures also shows that the supplements of gelsolin in animal models can decrease mortality and improve the damage of hyperoxia, burn, and sepsis conditions. ${ }^{12}$ Previous studies showed that GSN plays an important role in osteoclasts. Its deficiency blocks assembly and motility of prosoma in mouse osteoclasts, reduces bone resorption in vivo and produce increased bone mass and strength in mice. ${ }^{35,36}$ However, overexpression of GSN in the NK lymphoma cell line YTS has been reported to increase apoptosis and decrease cell proliferation and invasion. ${ }^{37}$ GSN is widely expressed in various cancers, Alzheimer's disease (AD), rheumatoid arthritis, and multiple disease. A review paper showed that patients with low GSN expression had significantly higher 5-year recurrence-free survival (RFS) rates than those with GSN overexpression. ${ }^{38}$ In addition, a study found that compared with healthy controls, patients with reduced plasma GSN levels had higher mortality rates, longer Hospital stays, and longer ventilation periods in the intensive care unit. It is found that the plasma GSN levels of patients who have recovered from the disease are increasing. ${ }^{39}$ Thus, we can see that the change of GSN will affect the emergence and progression of disease, no matter the expression level or sequence changing. It is really 
Table 2 Correlations Between GSN and Related Genes and Markers of Immune Cells

\begin{tabular}{|c|c|c|c|c|c|}
\hline \multirow[t]{2}{*}{ Description } & \multirow[t]{2}{*}{ Gene Markers } & \multicolumn{2}{|l|}{ None } & \multicolumn{2}{|l|}{ Purity } \\
\hline & & Cor & P-value & Cor & P-value \\
\hline \multirow[t]{2}{*}{ CD8+ T cell } & CD8A & 0.246 & $* * *$ & 0.244 & $* * *$ \\
\hline & CD8B & 0.117 & $*$ & 0.117 & $* * *$ \\
\hline \multirow[t]{3}{*}{$\mathbf{T}$ cell } & CD3D & 0.179 & $* * *$ & 0.17 & $* * *$ \\
\hline & CD3E & 0.22 & $* * *$ & 0.217 & $* * *$ \\
\hline & $\mathrm{CD} 2$ & 0.192 & $* * *$ & 0.185 & $* * *$ \\
\hline \multirow[t]{2}{*}{ B cell } & CDI9 & 0.306 & $* * *$ & 0.306 & $* * *$ \\
\hline & CD79A & 0.303 & $* * *$ & 0.29 & $* * *$ \\
\hline \multirow[t]{2}{*}{ Monocyte } & CD86 & 0.232 & $* * *$ & 0.218 & $* * *$ \\
\hline & CDII5 (CSFIR) & 0.453 & $0 \mathrm{e}+00$ & 0.439 & $* * *$ \\
\hline \multirow[t]{3}{*}{ TAM } & $\mathrm{CCL} 2$ & 0.363 & $* * *$ & 0.345 & $* * *$ \\
\hline & CD68 & 0.202 & $* * *$ & 0.186 & $* * *$ \\
\hline & ILIO & 0.295 & $* * *$ & 0.282 & $* * *$ \\
\hline \multirow[t]{3}{*}{ MI Macrophage } & INOS (NOS2) & -0.091 & & -0.082 & $1.12 \mathrm{e}-0 \mid$ \\
\hline & IRF5 & 0.343 & $* * *$ & 0.337 & $* * *$ \\
\hline & COX2(PTGS2) & 0.093 & & 0.071 & $1.67 \mathrm{e}-0 \mid$ \\
\hline \multirow[t]{3}{*}{ M2 Macrophage } & CDI63 & 0.34 & $* * *$ & 0.324 & $* * *$ \\
\hline & VSIG4 & 0.355 & $* * *$ & 0.355 & $* * *$ \\
\hline & MS4A4A & 0.356 & $* * *$ & 0.34 & $* * *$ \\
\hline \multirow[t]{3}{*}{ Neutrophils } & CD66b (CEACAM8) & -0.018 & $7.18 \mathrm{e}-01$ & 0 & $1.00 \mathrm{e}+00$ \\
\hline & CDIIb (ITGAM) & 0.403 & $0 e+00$ & 0.391 & $* * *$ \\
\hline & CCR7 & 0.456 & $0 \mathrm{e}+00$ & 0.462 & $* * *$ \\
\hline \multirow[t]{7}{*}{ Natural killer cell } & KIR2DLI & 0.005 & $9.18 \mathrm{e}-01$ & 0.015 & $7.66 \mathrm{e}-01$ \\
\hline & KIR2DL3 & -0.062 & $2.08 \mathrm{e}-0 \mathrm{I}$ & -0.073 & $1.55 \mathrm{e}-0 \mid$ \\
\hline & KIR2DL4 & -0.124 & $*$ & -0.13 & $*$ \\
\hline & KIR3DLI & 0.047 & $3.43 \mathrm{e}-0 \mathrm{I}$ & 0.052 & $3.17 \mathrm{e}-0 \mid$ \\
\hline & KIR3DL2 & 0.019 & $6.93 \mathrm{e}-0 \mathrm{I}$ & 0.03 & $5.57 \mathrm{e}-0 \mathrm{I}$ \\
\hline & KIR3DL3 & -0.092 & & -0.064 & $2.14 \mathrm{e}-0 \mid$ \\
\hline & KIR2DS4 & -0.031 & $5.35 \mathrm{e}-0 \mathrm{I}$ & -0.026 & $6.08 \mathrm{e}-01$ \\
\hline \multirow[t]{7}{*}{ Dendritic cell } & HLA-DPBI & 0.263 & $6 e-08$ & 0.251 & $7.56 \mathrm{e}-01$ \\
\hline & HLA-DQBI & 0.145 & $* *$ & 0.129 & $*$ \\
\hline & HLA-DRA & 0.154 & $* *$ & 0.141 & $* *$ \\
\hline & HLA-DPAI & 0.0198 & $* * *$ & 0.185 & $* * *$ \\
\hline & BDCA-I (CDIC) & 0.515 & $* * *$ & 0.54 & $* * *$ \\
\hline & BDCA-4(NRPI) & 0.542 & $0 e+00$ & 0.518 & $* * *$ \\
\hline & CDIIc (ITGAX) & 0.32 & $* * *$ & 0.304 & $* * *$ \\
\hline \multirow[t]{5}{*}{ ThI } & T-bet (TBX2I) & 0.216 & $* * *$ & 0.221 & $* * *$ \\
\hline & STAT4 & 0.268 & $* * *$ & 0.27 & $* * *$ \\
\hline & STATI & 0.012 & $8.1 \mathrm{le}-01$ & 0.024 & $6.47 \mathrm{e}-01$ \\
\hline & IFN- $\gamma($ IFNG) & -0.128 & $* *$ & -0.124 & $* *$ \\
\hline & TNF- $\alpha$ (TNF) & 0.08 & $1.05 \mathrm{e}-0 \mid$ & 0.054 & $2.91 \mathrm{e}-0 \mid$ \\
\hline \multirow[t]{4}{*}{ Th2 } & GATA3 & 0.364 & $2.39 \mathrm{e}-14$ & 0.376 & $* * *$ \\
\hline & STAT6 & 0.319 & $2.87 \mathrm{e}-11$ & 0.321 & $* * *$ \\
\hline & STAT5A & 0.418 & $5.1 e-19$ & 0.415 & $* * *$ \\
\hline & ILI3 & 0.075 & $1.26 \mathrm{e}-0 \mid$ & 0.089 & \\
\hline \multirow[t]{2}{*}{ Tfh } & $\mathrm{BCL6}$ & 0.549 & $4.65 e-34$ & 0.532 & $* * *$ \\
\hline & $\mid \mathrm{IL} 2 \mathrm{I}$ & 0.027 & $5.8 \mathrm{e}-01$ & 0.027 & $6.02 \mathrm{e}-01$ \\
\hline \multirow[t]{2}{*}{ ThI7 } & STAT3 & 0.422 & $0 e+00$ & 0.406 & $* * *$ \\
\hline & ILI7A & -0.249 & $2.86 \mathrm{e}-07$ & -0.264 & $* * *$ \\
\hline \multirow[t]{4}{*}{ Treg } & FOXP3 & 0.203 & $3.18 \mathrm{e}-05$ & 0.198 & $* * *$ \\
\hline & CCR8 & 0.277 & $9.61 \mathrm{e}-09$ & 0.271 & $* * *$ \\
\hline & STAT5B & 0.563 & $0 e+00$ & 0.542 & $* * *$ \\
\hline & TGF $\beta$ (TGFBI) & 0.598 & $|.32 \mathrm{e}-4|$ & 0.595 & $* * *$ \\
\hline
\end{tabular}


Table 2 (Continued).

\begin{tabular}{|l|l|l|l|l|l|}
\hline \multirow{2}{*}{ Description } & Gene Markers & \multicolumn{2}{l|}{ None } & \multicolumn{2}{l|}{ Purity } \\
\cline { 3 - 6 } & & Cor & P-value & Cor & P-value \\
\hline T cell exhaustion & PD-I (PDCDI) & 0.195 & $6.76 \mathrm{e}-05$ & 0.195 & $* * *$ \\
& CTLA4 & 0.061 & $2.16 \mathrm{e}-01$ & 0.049 & $3.41 \mathrm{e}-01$ \\
& LAG3 & 0.07 & $1.53 \mathrm{e}-01$ & 0.071 & $1.66 \mathrm{e}-01$ \\
& TIM-3 (HAVCR2) & 0.241 & $7.2 \mathrm{e}-07$ & 0.233 & $* * *$ \\
& GZMB & -0.125 & $*$ & -0.144 & $* *$ \\
\hline
\end{tabular}

Note: P-value significant codes: $0 \leq * * * \leq 0.001 \leq * * \leq 0.01 \leq * \leq 0.05$.

a target with broad implications and developing novel therapeutic strategy.

\section{Conclusions}

In conclusion, our result is consistent with those reported that GSN may be a prognostic biomarker in gastric cancer, and the value of GSN in immune infiltrates is potential. Although all data just from online databases analysis and qRT-PCR results, the evidence still have highly authoritative and quite match expectations. That is, the expression of GSN was exactly downregulate and this change was closely related to various prognostic value in $\mathrm{GC}$, especially the relevant knowledge

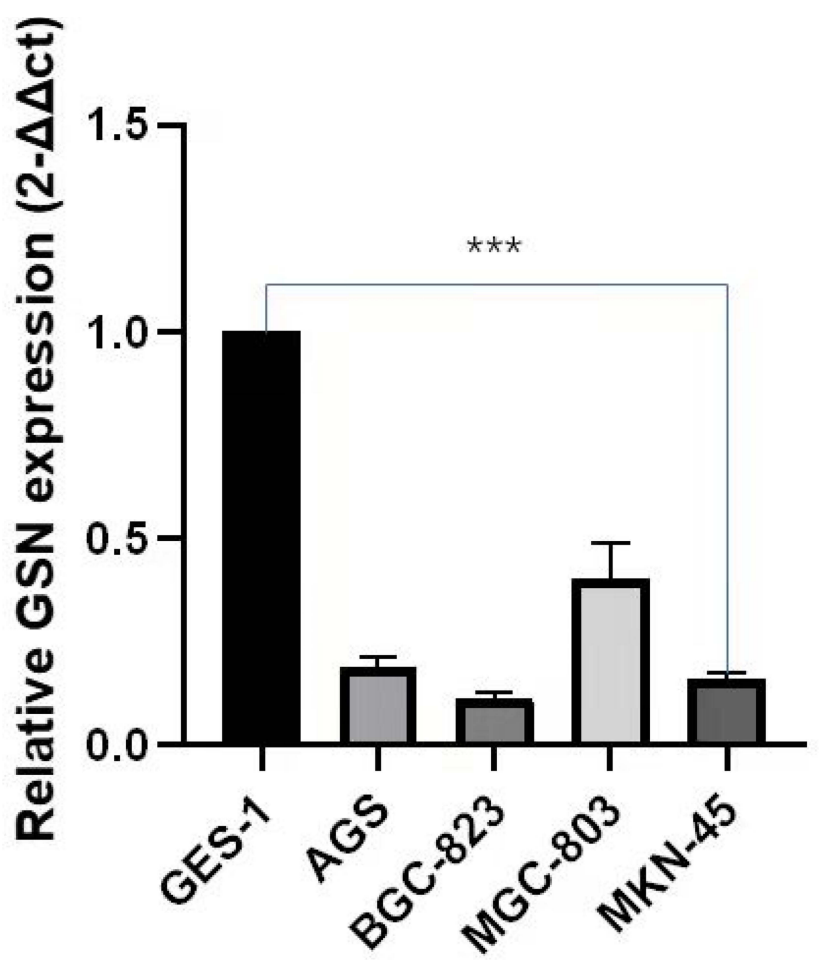

Figure 5 qRT-PCR of GSN expression in STAD cell lines and human normal gastric epithelial cell line GES-I. P-value significant codes: $p \leq * * * \leq 0.001$. about the immune features. In that respect, we really believe GSN can open-up huge possibilities in gastric cancer.

\section{Abbreviations}

GSN, gelsolin; GC, gastric cancer; STAD, stomach adenocarcinoma; OS, overall survival; FP, first progression; PPS, post progression survival; TAM, tumor-associatedmacrophage; TME, tumor microenvironment; DCs, dendritic cells; APC, antigen presenting cells.

\section{Data Sharing Statement}

The datasets set generated and/or analyzed during the current study are available in the TIMER (https://cis trome.shinyapps.io/timer/) and the human protein atlas (https://www.proteinatlas.org/) and Oncomine (https:// www.oncomine.org/resource/login.html) repository.

\section{Ethics Approval and Informed Consent}

All data for this study are derived from publicly available databases, and the Institutional Review Board of The First Affiliated Hospital, Guangzhou University of Chinese Medicine has provided the confirmation and waived the need for ethical approval.

\section{Consent for Publication}

All authors have made significant contributions to the concept and design, data acquisition or data analysis and interpretation; participated in drafting articles or critically revised important knowledge content; finally approved the version to be published; and agreed to be responsible for all aspects of the work.

\section{Author Contributions}

All authors participated in the research design, acquisition and data analysis, writing and revising the manuscript, and 
reached a consensus on the journal to which the article was submitted, approved the final version for publication, and agreed to be responsible for all the content of the article.

\section{Funding}

In particular, this study was supported by High-level hospital construction-Construction of a clinical-scientific integrated molecular diagnostic platform based on multidisciplinary development (2110300401); Chuang Xin Qiang Yuan Project (2016QN08); and Guangdong Medical Science and Technology Research Fund (B2020202).

\section{Disclosure}

The authors report no conflicts of interest related to this paper.

\section{References}

1. Arnold M, Abnet CC, Neale RE, et al. Global burden of 5 major types of gastrointestinal cancer. Gastroenterology. 2020;159(1):335349.e15. doi:10.1053/j.gastro.2020.02.068

2. Bray F, Ferlay J, Soerjomataram I, Siegel RL, Torre LA, Jemal A. Global cancer statistics 2018: GLOBOCAN estimates of incidence and mortality worldwide for 36 cancers in 185 countries [published correction appears in CA Cancer J Clin. 2020 Jul; 70(4):313]. CA Cancer J Clin. 2018;68(6):394-424. doi:10.3322/caac.21492

3. Thrift AP, El-Serag HB. Burden of gastric cancer. Clin Gastroenterol Hepatol. 2020;18(3):534-542. doi:10.1016/j.cgh.2019.07.045

4. GBD 2017 Stomach Cancer Collaborators. The global, regional, and national burden of stomach cancer in 195 countries, 1990-2017: a systematic analysis for the Global Burden of Disease study 2017 [published correction appears in Lancet Gastroenterol Hepatol. 2020 Mar;5(3):e2]. Lancet Gastroenterol Hepatol. 2020;5(1):42-54. doi:10.1016/S2468-1253(19)30328-0

5. Liu XY, Zhang J. A protocol of systematic review and meta-analysis of narrow band imaging endoscopy in detection of early gastric cancer. Medicine. 2020;99(29):e21269. doi:10.1097/ MD.0000000000021269

6. Allemani C, Matsuda T, Di Carlo V, et al. Global surveillance of trends in cancer survival 2000-14 (Concord-3): analysis of individual records for 37513025 patients diagnosed with one of 18 cancers from 322 population-based registries in 71 countries. Lancet. 2018;391(10125):1023-1075. doi:10.1016/S0140-6736(17)33326-3

7. Arnold M, Rutherford MJ, Bardot A, et al. Progress in cancer survival, mortality, and incidence in seven high-income countries 1995-2014 (ICBP SURVMARK-2): a population-based study. Lancet Oncol. 2019;20(11):1493-1505. doi:10.1016/S1470-2045(19)30456-5

8. Bai R, Chen N, Liang $T$, et al. Novel frontiers of treatment for advanced gastric or Gastroesophageal Junction Cancer (GC/GEJC) Will immunotherapy be a future direction? Front Oncol. 2020;10:912. doi:10.3389/fonc.2020.00912

9. Yin HL, Stossel TP. Control of cytoplasmic actin gel-sol transformation by gelsolin, a calcium-dependent regulatory protein. Nature. 1979;281(5732):583-586. doi:10.1038/281583a0

10. McGough AM, Staiger CJ, Min JK, Simonetti KD. The gelsolin family of actin regulatory proteins: modular structures, versatile functions. FEBS Lett. 2003;552(2-3):75-81. doi:10.1016/S00145793(03)00932-3
11. Müllauer L, Fujita H, Ishizaki A, Kuzumaki N. Tumor-suppressive function of mutated gelsolin in ras-transformed cells. Oncogene. 1993;8(9):2531-2536.

12. Yeh YL, Hu WS, Ting WJ, et al. Erratum to: hypoxia augments increased HIF-1a and reduced survival protein p-Akt in Gelsolin (GSN)-dependent cardiomyoblast cell apoptosis. Cell Biochem Biophys. 2018;76(1-2):323. doi:10.1007/s12013-017-0819-0

13. Zhuo J, Tan EH, Yan B, et al. Gelsolin induces colorectal tumor cell invasion via modulation of the urokinase-type plasminogen activator cascade. PLoS One. 2012;7(8):e43594. doi:10.1371/journal. pone. 0043594

14. Kelley DZ, Flam EL, Guo T, et al. Functional characterization of alternatively spliced GSN in head and neck squamous cell carcinoma. Transl Res. 2018;202:109-119. doi:10.1016/j.trsl.2018.07.007

15. Zhang R, Shang F, Li D, Zhang Y, Yuan L. The first Chinese renal gelsolin amyloidosis with the p.Asp174Asn mutation in the GSN gene: nephrology picture. $J$ Nephrol. 2021;34(4):1257-1259. doi:10.1007/s40620-020-00873-3

16. Mullany S, Souzeau E, Klebe S, et al. A novel GSN variant outside the G2 calcium-binding domain associated with Amyloidosis of the Finnish type. Hum Mutat. 2021;42(7):818-826. doi:10.1002/humu.24214

17. Oncomine 3.0.org [homepage on the Internet]. Michigan: genes, pathways, and networks in a collection of 18,000 cancer gene expression profiles; 2007. Available from: https://www.oncomine.org/ resource/login.html/. Accessed April 29, 2020.

18. The human protein atlas.org [homepage on the Internet]. Sweden: a pathology atlas of the human cancer transcriptome. Science; 2017. Available from: https://www.proteinatlas.org/. Accessed April 29, 2020.

19. TIMER.org [homepage on the Internet]. China: a web server for comprehensive analysis of tumor-infiltrating immune cells. Cancer Res. 2017. Available from: https://cistrome.shinyapps.io/timer/. Accessed April 29, 2020.

20. UALCAN.org [homepage on the Internet]. A portal for facilitating tumor subgroup gene expression and survival analyses. Neoplasia; 2017. Available from: http://ualcan.path.uab.edu/. Accessed April 29, 2020.

21. Szász AM, Lánczky A, Nagy Á, et al. Cross-validation of survival associated biomarkers in gastric cancer using transcriptomic data of 1065 patients. Oncotarget. 2016;7(31):49322-49333. doi:10.18632/ oncotarget. 10337

22. Nie $\mathrm{S}$, Yuan $\mathrm{Y}$. The role of gastric mucosal immunity in gastric diseases. J Immunol Res. 2020;2020:7927054. doi:10.1155/2020/7927054

23. Ahluwalia B, Magnusson MK, Öhman L. Mucosal immune system of the gastrointestinal tract: maintaining balance between the good and the bad. Scand J Gastroenterol. 2017;52(11):1185-1193. doi:10. 1080/00365521.2017.1349173

24. Zhang B, Ren J-L. Research progress of gastric mucosal immune mechanism. World Chin J Digestol. 2005;13(21):2605-2609. in Chinese.

25. Bimczok D, Kao JY, Zhang M, et al. Human gastric epithelial cells contribute to gastric immune regulation by providing retinoic acid to dendritic cells. Mucosal Immunol. 2015;8(3):533-544. doi:10.1038/ mi.2014.86

26. Bimczok D, Clements RH, Waites KB, et al. Human primary gastric dendritic cells induce a Th1 response to H. pylori. Mucosal Immunol. 2010;3(3):260-269. doi:10.1038/mi.2010.10

27. Tsujimura H, Tamura T, Kong HJ, et al. Toll-like receptor 9 signaling activates NF-kappaB through IFN regulatory factor-8/IFN consensus sequence binding protein in dendritic cells. J Immunol. 2004;172 (11):6820-6827. doi:10.4049/jimmunol.172.11.6820

28. Re F, Strominger JL. Toll-like receptor 2 (TLR2) and TLR4 differentially activate human dendritic cells. J Biol Chem. 2001;276 (40):37692-37699. doi:10.1074/jbc.M105927200

29. Bimczok D, Grams JM, Stahl RD, Waites KB, Smythies LE, Smith PD. Stromal regulation of human gastric dendritic cells restricts the Th1 response to Helicobacter pylori. Gastroenterology. 2011;141(3):929-938. doi:10.1053/j.gastro.2011.06.006 
30. Yuan C, Zhang E, Huang L, Wang J, Yang Q. Oral administration of inactivated porcine epidemic diarrhea virus activate DCs in porcine Peyer's patches [published correction appears in BMC Vet Res. 2018 Oct 3;14(1):303]. BMC Vet Res. 2018;14(1):239. doi:10.1186/ s12917-018-1568-z

31. Martínez-López JL, Torres J, Camorlinga-Ponce M, Mantilla A, Leal YA, Fuentes-Pananá EM. Evidence of Epstein-Barr virus association with gastric cancer and non-atrophic gastritis. Viruses. 2014;6 (1):301-318. doi:10.3390/v6010301

32. Hu WS, Ho TJ, Pai P, et al. Gelsolin (GSN) induces cardiomyocyte hypertrophy and BNP expression via p38 signaling and GATA-4 transcriptional factor activation. Mol Cell Biochem. 2014;390(12):263-270. doi:10.1007/s11010-014-1977-7

33. Fu C, Jiang A. Dendritic Cells and CD8 T cell immunity in tumor microenvironment. Front Immunol. 2018;9:3059. doi:10.3389/ fimmu.2018.03059

34. Li GH, Shi Y, Chen Y, et al. Gelsolin regulates cardiac remodeling after myocardial infarction through DNase I-mediated apoptosis. Circ Res. 2009;104(7):896-904. doi:10.1161/CIRCRESAHA.108.172882

35. Silacci P, Mazzolai L, Gauci C, Stergiopulos N, Yin HL, Hayoz D. Gelsolin superfamily proteins: key regulators of cellular functions. Cell Mol Life Sci. 2004;61(19-20):2614-2623. doi:10.1007/s00018004-4225-6
36. Chellaiah M, Kizer N, Silva M, Alvarez U, Kwiatkowski D, Hruska KA. Gelsolin deficiency blocks podosome assembly and produces increased bone mass and strength. J Cell Biol. 2000;148 (4):665-678. doi:10.1083/jcb.148.4.665

37. Ma X, Sun W, Shen J, et al. Gelsolin promotes cell growth and invasion through the upregulation of p-AKT and p-P38 pathway in osteosarcoma. Tumour Biol. 2016;37(6):7165-7174. doi:10.1007/ s13277-015-4565-X

38. Kim JC, Ha YJ, Tak KH, et al. Opposite functions of GSN and OAS2 on colorectal cancer metastasis, mediating perineural and lymphovascular invasion, respectively. PLoS One. 2018;13(8):e0202856. doi:10.1371/journal.pone.0202856

39. Peddada N, Sagar A, Ashish A, Garg R. Plasma gelsolin: a general prognostic marker of health. Med Hypotheses. 2012;78(2):203-210. doi:10.1016/j.mehy.2011.10.024

\section{Publish your work in this journal}

The International Journal of General Medicine is an international, peer-reviewed open-access journal that focuses on general and internal medicine, pathogenesis, epidemiology, diagnosis, monitoring and treatment protocols. The journal is characterized by the rapid reporting of reviews, original research and clinical studies across all disease areas. The manuscript management system is completely online and includes a very quick and fair peer-review system, which is all easy to use. Visit http://www.dovepress.com/ testimonials.php to read real quotes from published authors. 